\title{
Vacunación contra COVID-19 en niños
}

\author{
COVID-19 vaccination in children
}

\author{
Ranferi Aragón-Nogales, * María Guadalupe Miranda-Novales ${ }^{\ddagger}$ \\ * Departamento de Infectología, UMAE Hospital de Pediatría del Centro Médico Nacional, Siglo XXI; ${ }^{\text {Unidad }}$ \\ de Investigación en Análisis y Síntesis de la Evidencia, Instituto Mexicano del Seguro Social. México.
}

\section{INTRODUCCIÓN}

La pandemia actual ocasionada por el coronavirus del síndrome respiratorio agudo severo tipo 2 (SARSCoV-2), causante de la enfermedad denominada COVID-19 (coronavirus disease), ha infectado a más de 210 millones de habitantes y causado más de 4.5 millones de muertes hasta finales de agosto de 2021. Lo anterior corresponde a una tasa de letalidad global aproximada de $2 \%$ de acuerdo a registros de la Organización Mundial de la Salud (OMS). ${ }^{1}$

Desde el inicio de la pandemia, el grupo pediátrico presentó menor cantidad de casos graves, hospitalizaciones y muertes, comparado con lo observado en adultos, reportando tasas de prevalencia variables de acuerdo a cada región. ${ }^{2}$ Sin embargo, en países como Estados Unidos de América (EUA), en los meses más recientes, se ha registrado un incremento en el porcentaje de casos. Para agosto 26 de 2021, se habían acumulado 4.8 millones de casos en niños, con un incremento exponencial en el último mes de cinco veces más con respecto a los registros previos. Así, los niños representaron $22.4 \%$ de los casos reportados a la semana, con un incremento de $7 \%$ durante las últimas dos semanas. ${ }^{3}$ En México, al 22 de agosto de 2021 se han acumulado 156,990 casos en niños. El grupo más afectado es el de 12 a 17 años, que concentra $59.6 \%$ de los casos, y en el que han ocurrido 758 defunciones. ${ }^{4}$
Desde abril de 2020, se estableció el Acelerador de Acceso a Herramientas COVID-19 (ACT) como una colaboración para el desarrollo, producción y acceso equitativo a las pruebas, tratamientos y vacunas COVID-19. ${ }^{5}$ De acuerdo con el rastreador interactivo en línea desarrollado por el Centro de Vacunas de la Escuela de Higiene y Medicina Tropical de Londres, para agosto de 2021, hay más de 300 vacunas candidatas, con 103 en estudios clínicos. Lo anterior refleja de manera notable los avances en cuanto a las plataformas de vacunas que previamente no se habían autorizado, como las moléculas de ARN mensajero o moléculas de $\mathrm{ADN}{ }^{6,7}$

El Grupo de Expertos de Asesoramiento Estratégico sobre Inmunización (SAGE) de la OMS emite recomendaciones sobre la aprobación de las vacunas que han demostrado ser seguras y eficaces. Este organismo establece una guía para la vacunación de grupos prioritarios, de acuerdo con la disponibilidad y tomando en cuenta diferentes contextos epidemiológicos, pero reconociendo que, en las etapas iniciales, el objetivo es la reducción de la morbilidad y mortalidad por COVID-19. ${ }^{7,8}$ Con esta guía, cada país establece sus propios grupos técnicos asesores de inmunizaciones (GTAV), a fin de adaptar y analizar las recomendaciones a sus programas nacionales, de acuerdo con las características de cada región. ${ }^{9}$ Desde el inicio de la vacunación, hemos sido testigos de que existe un suministro limitado, lo cual supera ampliamente a la demanda,

Correspondencia: María Guadalupe Miranda-Novales, E-mail: guadalupe.mirandan@imss.gob.mx

Citar como: Aragón-Nogales R, Miranda-Novales MG. Vacunación contra COVID-19 en niños. Rev Mex Pediatr. 2021; 88(3): 89-92. https:// dx.doi.org/10.35366/102187 
pero en cualquier país o región la prioridad es vacunar a las personas con mayor riesgo de enfermedad grave y muerte. Por esta razón, y dado que los niños se han considerado un grupo de bajo riesgo, se han quedado como meta secundaria para vacunar, hasta garantizar la seguridad de la población de mayor riesgo. ${ }^{8}$

\section{VACUNACIÓN DE COVID-19 EN NIÑOS}

En los niños, el impacto de la pandemia de COVID-19 ha sido importante en su educación, salud y su bienestar general. Si bien la mayoría presenta un cuadro clínico leve o asintomático, contribuyen a la transmisión comunitaria, con implicaciones para el control de la pandemia. ${ }^{10}$

El 11 de diciembre de 2020, la Administración de Alimentos y Medicamentos (FDA, por sus siglas en inglés) de EUA emitió una autorización de uso de emergencia de la vacuna BNT162b2 de Pfizer/BioNTech para personas mayores de 16 años; después, el 31 de diciembre de 2020, la OMS hizo lo mismo, al ser la primera vacuna que incluyó en la lista de uso de emergencia. ${ }^{8,11}$

A pesar de los grandes avances alcanzados con los diversos tipos de vacunas, aún hay ensayos clínicos aleatorizados (ECA) limitados en niños como mencionaremos a continuación:

La vacuna BNT162b2 (Pfizer/BioNTech), que contiene ARN mensajero modificado con nucleósidos que codifica la glucoproteína Spike del SARS-CoV-2, fue evaluada en adolescentes de 12 a 15 años mediante un ECA. En ese estudio se compararon 1,131 participantes, quienes recibieron un régimen de dos dosis, administrado con 21 días de diferencia, con 1,129 que recibieron placebo. En los resultados se demostró que la vacuna era inmunogénica, al contrastarse con lo ocurrido con el grupo de edad 16 a 25 años, pero se encontró mayor respuesta en los adolescentes. La eficacia reportada fue de 100\% (intervalo de confianza IC $95 \%, 75.3$ a $100 \%$ ) a partir de los siete días de la segunda dosis, destacando que no se reportaron casos de COVID-19 grave. Los eventos adversos fueron de corta duración, siendo más frecuentes después de la segunda dosis. El dolor en el sitio de la inyección fue la reacción local más común (IC 95\% 79 a 86\%) y los eventos sistémicos más frecuentes fueron cefalea (IC $95 \% 55$ a $65 \%$ ) y fatiga (IC $95 \% 60$ a $66 \%$ ). No hubo eventos adversos graves relacionados con la vacuna. ${ }^{12}$

La vacuna de virus inactivados de SARS-CoV-2, CoronaVac, fue probada mediante ensayo clínico, controlado, aleatorizado, doble ciego en niños y ado- lescentes de tres a 17 años, con 550 participantes en tres grupos: grupo 1 con 219 participantes, recibió una dosis de $1.5 \mu \mathrm{g}$. El grupo 2, con 217 participantes, recibió dosis de $3 \mu \mathrm{g}$; mientras que el grupo 3, con 114 participantes, recibió hidróxido de aluminio. Cada grupo recibió dos dosis con una diferencia de 28 días. La vacuna demostró ser inmunogénica, con tasas de seroconversión de anticuerpos neutralizantes superiores a $96 \%$ después de la segunda dosis, sin observar diferencias significativas entre grupos de edad 3-5, 6-11 y 12-17 años. También demostró ser segura, al reportar únicamente reacciones de intensidad leve a moderada, siendo transitorias. Se reportó al menos una reacción adversa dentro de los 28 días de cualquiera de la dosis en 146 (27\%) de 550 participantes; la mayoría ocurrió dentro de los primeros siete días. El dolor en el lugar de la inyección fue el síntoma más informado en 73 (13\%), seguido de fiebre en 25 (5\%), destacando que ninguno de los efectos adversos graves fue relacionado con la vacunación. ${ }^{13}$

La vacuna ARNm-1273 (Moderna) es una dispersión líquida de ARN mensajero que codifica la glicoproteína S de SARS-CoV-2. Esta vacuna se evaluó en adolescentes de 12 a 17 años, en un ECA: 2,489 participantes recibieron la vacuna dos dosis, con diferencia de 28 días, y 1,243 recibieron placebo. Se reportó respuesta serológica de $98.8 \%$, cumpliendo criterios de no inferioridad comparado con el grupo de adultos jóvenes. Después de 14 días de la segunda dosis, la eficacia fue de 93\%. En el grupo que recibió la vacuna, las reacciones adversas que se reportaron después de la primera o segunda dosis fueron dolor en el lugar de la inyección (93.1 y 92.4\%, respectivamente), cefalea (44.6 y $70.2 \%$ ) y fatiga ( 47.9 y $67.8 \%$, respectivamente), pero sin observarse eventos adversos graves relacionados a la vacuna. ${ }^{14}$

\section{AVANCES DE VACUNACIÓN EN NIÑOS Y ADOLESCENTES}

La OMS ha aprobado el uso de emergencia de al menos 13 vacunas distintas a través de cuatro plataformas para su aplicación en la población adulta; sin embargo, hasta ahora, para la edad pediátrica únicamente ha recomendado el uso de la vacuna BNT162b2 (Pfizer/ BioNTech).

Por su parte, Canadá el 5 de mayo de 2021 fue el primer país en aprobar el uso de la vacuna BNT162b2 en adolescentes de 12 a 15 años; después, a finales del mismo mes, EUA y la Agencia Europea de Medicamentos hicieron lo mismo. 
El 8 de mayo de 2021 China aprobó la vacuna CoronaVac para su uso de emergencia en la población de 3-17 años; después, en Indonesia, se aprobó para la población de 12 a 17 años a finales de junio de $2021 .{ }^{15}$

El Reino Unido ha reportado durante su segunda ola de pandemia en menores de 18 años una tasa de hospitalización de 100 a 400 por millón, y una tasa de mortalidad de dos muertes por millón, la mayoría con enfermedades subyacentes. Por esta razón, recomienda la aplicación de la vacuna BNT162b2 a personas mayores de 12 años con condiciones clínicas subyacentes o contactos domésticos de personas en riesgo de desarrollar una enfermedad grave por COVID-19. ${ }^{16}$

\section{SEGURIDAD DE LAS VACUNAS CONTRA COVID-19 EN NIÑOS Y ADOLESCENTES}

Garantizar la seguridad de las vacunas es trascendental para la población general y, antes de emitir recomendaciones definitivas, se debe seguir un proceso riguroso de vigilancia tanto a corto como largo plazo. Los eventos adversos extremadamente raros, pero que pueden ser graves, pueden no identificarse en los ensayos clínicos iniciales, por lo que deben monitorizarse y notificarse para evaluar la asociación.

El primer informe de seguridad de la vacuna PfizerBioNTech en EUA se publicó recientemente. Al 16 de julio de 2021, en dicho país, aproximadamente $8.9 \mathrm{mi}$ llones de adolescentes de 12 a 17 años habían recibido la vacuna. Se utilizaron dos mecanismos de vigilancia; el primero fue el sistema de reportes de eventos adversos asociados a vacunación (VAERS) y el otro fue V-Safe (un sistema de vigilancia basado en el uso de teléfonos inteligentes). ${ }^{17}$ VAERS es un mecanismo de vigilancia pasivo, el cual recibe informes de médicos, fabricantes de vacunas o del público general. En este sistema se reportaron 9,246 informes; 5,376 (58.1\%) ocurrieron en adolescentes de 12 a 15 años y 3,780 (41.9\%) en personas de 16 a 17 años. En 8,383 (90.7\%) fueron eventos adversos no graves, los más frecuentes fueron mareos, síncope, náuseas y cefalea. De los 863 (9.3\%) de eventos adversos considerados graves, en 397 (4.3\%) fueron pacientes con miocarditis. ${ }^{17}$

Por su parte, V-Safe es un sistema activo basado en el uso de teléfonos inteligentes, en el cual se realiza un automonitoreo y, mediante encuestas en línea, registra la presencia de eventos adversos, en particular si se requiere atención médica. Con la participación de 129,059 adolescentes de 12 a 17 años, las reacciones notificadas con más frecuencia (después de cualquiera de las dosis) fueron: dolor en el sitio de la inyección, fatiga, cefalea y mialgia. Estas reacciones se registraron principalmente al día siguiente de la aplicación de la vacuna, siendo la mayor frecuencia de las reacciones sistémicas en la segunda dosis. En la semana siguiente después de la administración de la segunda dosis, un tercio refirió fiebre y un cuarto de los participantes reportaron incapacidad para realizar sus actividades. Menos de 1\% requirió atención médica después de cualquiera de las dosis, siendo un total de 56 adolescentes (0.04\%) que requirieron hospitalización. ${ }^{17}$

Con excepción de la miocarditis, a gran escala, los hallazgos iniciales de seguridad son similares a los descritos en los ECA. La miocarditis es un evento adverso grave poco común; sin embargo, después de un análisis de la evidencia disponible, el 23 de junio de 2021, el Comité Asesor sobre Prácticas de Inmunización continúa recomendando el uso de la vacuna en personas mayores de 12 años. Lo anterior debido a que el beneficio individual y poblacional supera el riesgo, pero se tiene que informar a la población que existe el riesgo de esta complicación, en especial en hombres de 12 a 29 años, por lo cual, se enfatiza la necesidad de buscar atención si presentan síntomas sugerentes de esta condición. ${ }^{18}$

\section{SITUACIÓN EN MÉXICO}

La estrategia de vacunación en México estima que para el 29 de agosto de 2021, aproximadamente 58\% de la población mayor de 18 años tiene un esquema completo. ${ }^{19}$ Sin embargo, aún no ha iniciado la vacunación en la población pediátrica. Pero el 11 de junio de 2021, la Comisión Federal para la Protección contra Riesgos Sanitarios (COFEPRIS) amplió el grupo etario a mayores de 12 años, para el uso de emergencia de la vacuna Pfizer/BioNTech. ${ }^{20}$

El $1^{\circ}$ de octubre de 2021 se inició en México el registro de los menores de 12 a 17 años con comorbilidades para que sean vacunados próximamente con vacuna Pfizer/BioNTech. Las comorbilidades o condiciones subyacentes incluyen: enfermedades cardiacas crónicas, enfermedad pulmonar crónica, afecciones crónicas de riñón, hígado o sistema digestivo, enfermedad neurológica crónica, enfermedades endocrinas, inmunosupresión, asplenia o disfunción del bazo y enfermedades hematológicas, anomalías genéticas graves que afectan a varios sistemas, así como adolescentes embarazadas.

\section{COMENTARIOS FINALES}

Ante la pandemia actual de COVID-19, en un tiempo sin precedentes, las vacunas han demostrado ser efi- 
caces y seguras, ya que se ha demostrado que reducen la enfermedad sintomática, hospitalización y muerte; no obstante, aún hay desafíos por enfrentar, como son la evasión inmunológica que pueden presentar las variantes, identificar los candidatos y tiempos ideales de refuerzos, así como acelerar la disponibilidad mundial.

Es muy probable que, conforme avance el programa de vacunación, se modificará la población susceptible, por lo que se puede incrementar el riesgo de infección por COVID-19, tanto en niños como en individuos no vacunados. Los médicos tenemos la responsabilidad de promover los beneficios de las vacunas ya existentes, a fin de acelerar los esquemas atrasados y enfatizar las medidas de prevención hasta que sea posible vacunar al grupo pediátrico.

\section{REFERENCIAS}

1. Weekly epidemiological update on COVID-19 - 17 August 2021 [Internet]. Available in: https://www.who.int/publications/m/item/ weekly-epidemiological-update-on-covid-19---17-august-2021

2. Borrelli M, Corcione A, Castellano F, Fiori Nastro F, Santamaria F. Coronavirus disease 2019 in children. Front Pediatr. 2021; 9: 668484.

3. Children and COVID-19: State-Level Data Report [Internet]. Available in: http://www.aap.org/en/pages/2019-novel-coronaviruscovid-19-infections/children-and-covid-19-state-level-data-report/

4. Datos abiertos. Dirección General de Epidemiología. Disponible en: https://www.gob.mx/salud/documentos/datos-abiertos-152127

5. The Access to COVID-19 Tools (ACT) Accelerator [Internet]. Available in: https://www.who.int/initiatives/act-accelerator

6. Shrotri M, Swinnen T, Kampmann B, Parker EPK. An interactive website tracking COVID-19 vaccine development. Lancet Glob Health. 2021; 9(5): e590-e592.

7. COVID-19 vaccine tracker[Internet]. Available in: https://vac-lshtm. shinyapps.io/ncov_vaccine_landscape/

8. Organización Mundial de la Salud. Hoja de ruta del SAGE de la OMS para el establecimiento de prioridades en el uso de vacunas contra la COVID-19 en un contexto de suministros limitados: conjunto de criterios para orientar la planificación y las recomendaciones posteriores en distintos contextos epidemiológicos y posibles situaciones de suministro de vacunas, 20 de octubre de 2020, Versión 1. Organización Mundial de la Salud; 2020. Disponible en: https://apps.who.int/ iris/handle/10665/341447

9. Enfermedad por el coronavirus (COVID-19): Vacunas [Internet]. Disponible en: https://www.who.int/es/news-room/q-a-detail/ coronavirus-disease-(covid-19)-vaccines
10. Covid-19 GTA de V. Priorización inicial y consecutiva para la vacunación contra SARS-CoV-2 en la población mexicana. Recomendaciones preliminares. Salud Pública Méx. 2021; 63(2): 288-309.

11. Kampmann B, Okomo U. COVID-19 vaccines for children in LMICs: another equity issue. Lancet (London, England) [Internet]. Available in: https://www.ncbi.nlm.nih.gov/pmc/articles/ PMC8324110/

12. Frenck RW, Klein NP, Kitchin N, Gurtman A, Absalon J, Lockhart $\mathrm{S}$, et al. Safety, Immunogenicity, and efficacy of the BNT162b2 Covid-19 vaccine in adolescents. N Engl J Med. 2021; 385: 239250.

13. Han B, Song Y, Li C, Yang W, Ma Q, Jiang Z, et al. Safety, tolerability, and immunogenicity of an inactivated SARS-CoV-2 vaccine (CoronaVac) in healthy children and adolescents: a double-blind, randomised, controlled, phase 1/2 clinical trial. Lancet Infect Dis. 2021; S1473-3099(21)00319-4.

14. Ali K, Berman G, Zhou H, Deng W, Faughnan V, CoronadoVoges $M$, et al. Evaluation of mRNA-1273 SARS-CoV-2 vaccine in adolescents. N Engl J Med. 2021. doi: 10.1056/ NEJMoa2109522.

15. Zou X, Cao B. COVID-19 vaccines for children younger than 12 years: are we ready? Lancet Infect Dis. 2021; S14733099(21)00384-4.

16. JCVI statement on COVID-19 vaccination of children and young people aged 12 to 17 years: 4 August 2021 [Internet]. GOV. UK. Available in: https://www.gov.uk/government/publications/ jcvi-statement-august-2021-covid-19-vaccination-of-children-andyoung-people-aged-12-to-17-years/jcvi-statement-on-covid-19vaccination-of-children-and-young-people-aged-12-to-17-years4-august-2021

17. Hause AM. COVID-19 vaccine safety in adolescents aged 12-17 Years - United States, December 14, 2020-July 16, 2021. MMWR Morb Mortal Wkly Rep [Internet]. 2021; 70. Available in: https:// www.cdc.gov/mmwr/volumes/70/wr/mm7031e1.htm

18. Gargano JW. Use of mRNA COVID-19 Vaccine after reports of myocarditis among vaccine recipients: update from the advisory committee on immunization practices - United States, June 2021. MMWR Morb Mortal Wkly Rep [Internet]. 2021; 70(27): 977-982. Available in: https://www.cdc.gov/mmwr/volumes/70/wr/ $\mathrm{mm} 7027 \mathrm{e} 2 . \mathrm{htm}$

19. Secretaría de Salud. Informes Epidemiológicos de la situación de COVID19 en México 2021 [Internet]. gob.mx. Disponible en: http:// www.gob.mx/salud/documentos/informes-epidemiologicos-de-lasituacion-de-covid19-en-mexico-2021?idiom=es

20. COFEPRIS emite modificación a la autorización para uso de emergencia de vacuna pfizer-biontech; permitirá aplicación a partir de 12 años [Internet]. gob.mx. Disponible en: http://www.gob.mx/ cofepris/articulos/cofepris-emite-modificacion-a-la-autorizacionpara-uso-de-emergencia-de-vacuna-pfizer-biontech-permitiraaplicacion-a-partir-de-12-anos?idiom=es 